\title{
Associations of complement factor B and complement component 2 genotypes with subtypes of polypoidal choroidal vasculopathy
}

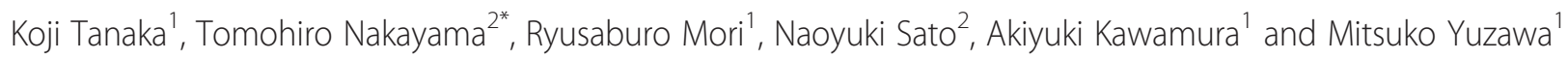

\begin{abstract}
Background: We previously reported on subtypes of polypoidal choroidal vasculopathy (PCV), and categorized PCV as polypoidal choroidal neovascularization (CNV) and typical PCV. The aim of this study was to clarify whether complement component 2 (C2) and complement factor B (CFB) genotypes are associated with subtypes of polypoidal choroidal vasculopathy, such as polypoidal CNV and typical PCV.

Methods: First, we categorized 677 patients into typical age-related macular degeneration (tAMD; 250 patients), PCV (376) and retinal angiomatous proliferation (RAP; 51). Second, we categorized 282 patients with PCV as having polypoidal CNV (84 patients) or typical PCV (198) based on indocyanine green angiographic findings. In total, 274 subjects without AMD, such as PCV and CNV, served as controls. A SNP (rs547154) in the C2 gene and three SNPs (rs541862, rs2072633, rs4151667) in the CFB gene were genotyped, and case-control studies were performed in subjects with these PCV subtypes.

Results: In tAMD, no SNPs were associated with allele distributions. In PCV, rs547154 and rs2072633 were associated with allele distributions. RAP was only associated with rs2072633. After logistic regression analysis with adjustment for confounding factors, tAMD, PCV and RAP were found to be associated with rs 2072633.

As to PCV subtypes, there were significant differences in the distributions of rs547154, rs541862 and rs2072633 in the case-control studies for polypoidal CNV, but not between the typical PCV and control groups. Logistic regression analysis with adjustment for confounding factors showed the distributions of rs547154, rs541862 and rs2072633 to differ significantly between the controls and polypoidal CNV cases and that these SNPs were protective. The AVA genotype of rs2072633 was significantly more common in the polypoidal CNV than in the typical PCV group $(p=0.03)$, even with adjustment for polyp number and greatest linear dimension.
\end{abstract}

Conclusions: PCV might be genetically divisible into polypoidal CNV and typical PCV. The C2 and CFB gene variants were shown to be associated with polypoidal CNV. Typical PCV was not associated with variants in these genes.

Keywords: Subtypes of PCV, C2, CFB, Genetic variants

\section{Background}

Age-related macular degeneration (AMD) is a leading cause of blindness in Western countries and its prevalence is increasing in Japan [1]. AMD is thought to be a heterogeneous multifactorial disease associated with several environmental factors and genetic variants. Hypertension [2] and cigarette smoking [3] are closely

\footnotetext{
* Correspondence: nakayama.tomohiro@nihon-u.ac.jp

${ }^{2}$ Department of Pathology and Microbiology, Nihon University School of Medicine, Tokyo, Japan

Full list of author information is available at the end of the article
}

related to the development of AMD. Identification of AMD susceptibility genes might increase our ability to predict the risk of developing this disease. Complement factor $\mathrm{H}(\mathrm{CFH})$, age-related maculopathy susceptibility 2 (ARMS2) and high-temperature requirement factor A1 (HTRA1) have been shown to be associated with AMD in both Japanese and Caucasian patients [4-7]. In addition, complement component $2(\mathrm{C} 2)$ and complement factor B (CFB) known as activators of alternative complement cascades are reportedly related to AMD in Caucasians [8]. Both were reported to be protective 
genes against AMD development [9,10]. Genetic studies of PCV have found no association between either $\mathrm{C} 2$ or CFB and PCV [11,12]. Nakata et al. reported that, in the Japanese population, $\mathrm{C} 2$ and $\mathrm{CFB}$ are associated with both PCV and typical AMD (tAMD) [13].

Polypoidal choroidal vasculopathy (PCV), characterized by a branching vascular network with polypoidal lesions detectable by indocyanine green angiography (IA) [14], is included among the forms of exudative AMD in Japan [15]. Our group previously reported on subtypes of PCV, and categorized PCV as polypoidal choroidal neovascularization $(\mathrm{CNV})$ and PCV in the narrow sense (also referred to as typical PCV) [16]. In the first type, both feeder and draining vessels are visible on IA and network vessels are numerous. This type is thought to be the representative form of $\mathrm{CNV}$ beneath the retinal pigment epithelium. In the second group, neither feeder nor draining vessels are detectable and the number of network vessels is small. This type is thought to represent an abnormality of the choroidal vasculature based on hyaline arteriosclerosis [17]. We also showed that there are differences in these two types classified according to IA and optic coherence tomography findings [18]. Genetically, we demonstrated an association between the ARMS2 gene and these two types of PCV [19]. There was a significant ARMS2 gene difference in case-control studies of polypoidal CNV, but no difference between the typical PCV and control groups. This observation suggests that PCV might be genetically divisible into polypoidal $\mathrm{CNV}$ and typical PCV.

The possibility of dividing PCV into two types has been raised by other investigators. Okubo et al. reported that PCV can be divided into two types; the small-short and large-long types, but the clinical features in their report differed from those described by our group [20]. Miki et al. recently advocated dividing PCV into polypoidal lesions with a clear branching vascular network and polypoidal lesions without such a vascular network [21]. After classifying PCV into two types based on IA findings, we conducted ARMS2 and $\mathrm{CFH}$ genotyping for our patients. The results were highly consistent with our report showing typical PCV to be unrelated to the ARMS2 gene.

The present study aimed to investigate whether there is an association between the $\mathrm{C} 2$ or the CFB gene and any of the subtypes of PCV. To our knowledge, this is the first study to examine associations of the $\mathrm{C} 2$ and $\mathrm{CFB}$ genes with PCV subtypes.

\section{Methods}

\section{Participants}

Six hundred and seventy-seven patients diagnosed as having AMD at Nihon University Surugadai Hospital in Tokyo were enrolled in this study between 2008 and 2010 (472 men, 205 women; mean age 72.11 years). We then categorized AMD as tAMD, PCV and RAP based on IA and color photograph. (tAMD; 187 men, 63 women, PCV; 266 men, 110 women, RAP;19 men, 32 women)

Furthermore, we also classified PCV patients into groups with two different types of PCV, polypoidal CNV and typical PCV. Two hundred and eighty-two (195 men, 87 women; mean age $70.0 \pm 8.8$ years) out of 376 patients were enrolled after classification based on whether or not both feeder and draining vessels were seen on IA (Figures 1 and 2). Due to unclear IA findings, we could not classify the remaining 94 patients. Eighty-four patients were diagnosed with polypoidal CNV, 198 with typical PCV. Polyp numbers and greatest linear dimension (GLD) were determined by IA at the first visit.

Information on hypertension, diabetes mellitus and smoking was obtained from medical histories collected for each patient. Smokers were defined as current or former smokers, whereas non-smokers were defined as subjects with no previous or current smoking history.

In total, 274 subjects free of AMD (110 men, 164 women; mean age $72.9 \pm 7.4$ years) served as controls. There were no remarkable findings on fundus examinations of the controls. Informed consent was obtained from each individual as per the protocol approved by the $\mathrm{Hu}$ man Studies Committee of Nihon University. This investigation was performed according to the guidelines of the Declaration of Helsinki.

\section{Genotyping}

DNA was extracted from peripheral blood leukocytes by the phenol and chloroform extraction method [22,23]. Genotyping was performed using the TaqMan ${ }^{\circ}$ SNP Genotyping Assay (Applied Biosystems Inc. Foster City,

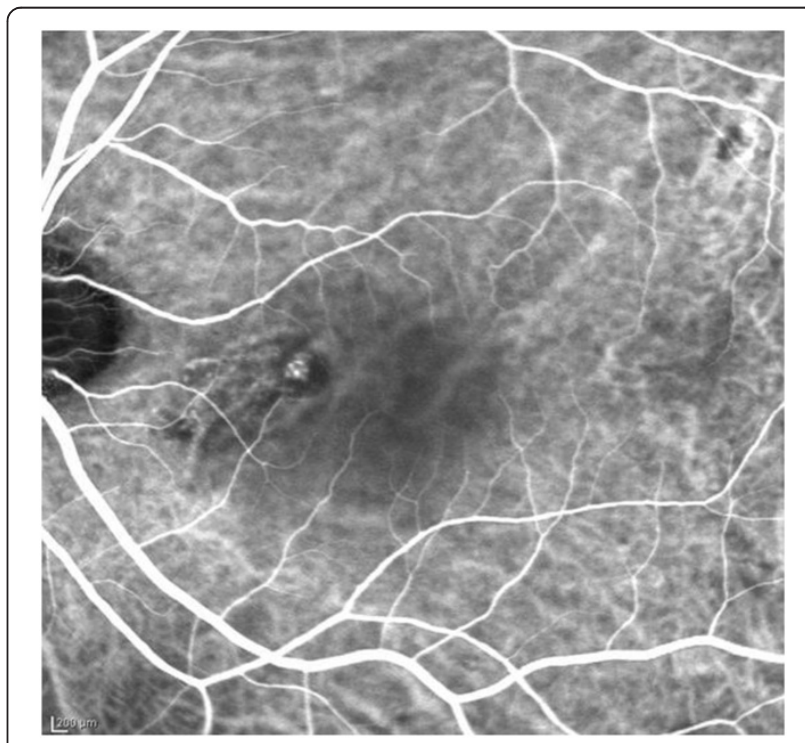

Figure 1 Typical PCV. Neither feeder nor draining vessels were visible in the early phase of indocyanine green angiography. The network is composed of a small number of vessels with a polypoidal lesion. 


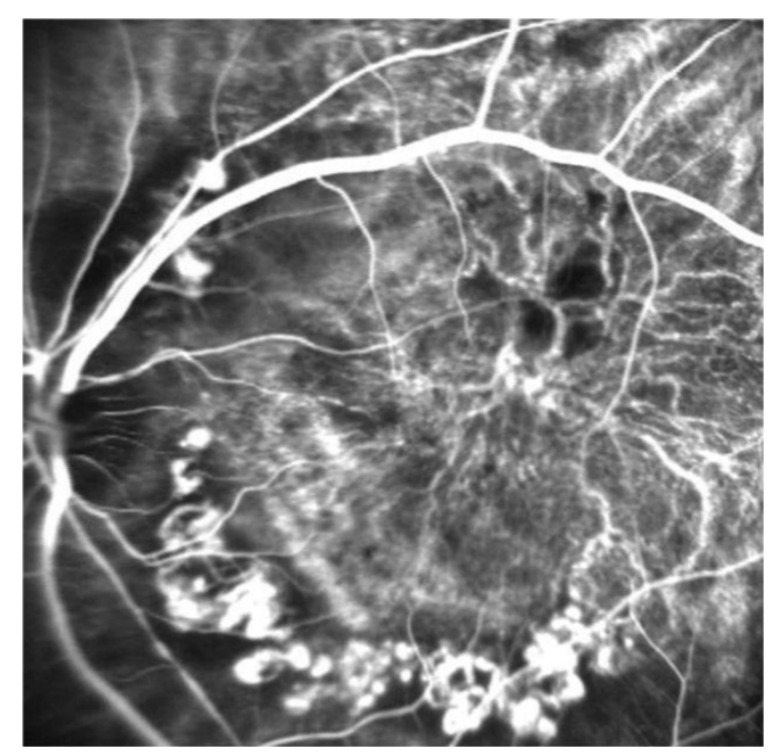

Figure 2 Polypoidal CNV. Both feeder and draining vessels were observed in the early phase of indocyanine green angiography. Large numbers of network vessels were seen to be fluorescing in an umbrella-like configuration. Several of the polypoidal lesions were dilatations of marginal tortuous vessels.

CA, USA). TaqMan ${ }^{\circ}$ SNP Genotyping Assays were performed using the Taq amplification method [22,23].

We targeted C2 rs547154(IVS10), and CFB rs541862, rs2072633(IVS17) and rs4151667(H9L), all of which were identified as having positive associations with AMD in prior studies [11,13].

Plates were read on the SDS 7700 instrument with the end-point analysis mode of the SDS version 1.6.3 software package (Applied Biosystems). Genotypes were determined visually based on the dyecomponent fluorescent emission data depicted in the X-Y scatter-plot of the SDS software. Genotypes were also determined automatically by the signal processing algorithms of the software [22,23].

\section{Statistical analysis}

Data are shown as means \pm SD. Differences between the PCV subtype and control groups were assessed by analysis of variance (ANOVA) followed by Fisher's protected least significant difference test. Hardy-Weinberg equilibrium was assessed by chi-squared analysis. The overall distribution of alleles was analyzed using $2 \times 2$ contingency tables. The distribution of the genotypes between patient groups and controls was tested using a 2-sided Fisher's exact test and multiple logistic regression analysis. After Bonferroni correction, statistical significance was set at $\mathrm{p}<0.0125$.

Based on the genotype data of the genetic variations, linkage disequilibrium (LD) analyses and a haplotypebased case-control study were carried out using the expectation maximization algorithm with the SNPAlyze software program ver3.2 (Dynacom, Yokohama, Japan). $\left|D^{\prime}\right|$ values $>0.5$ were used to assign SNP locations to one haplotype block. The frequency distribution of occurrence of the haplotypes was calculated by $\chi^{2}$ analyses.

\section{Results}

The clinical features of AMD patients and the control group are shown in Table 1. Distributions of genotypes and alleles are shown in Table 2. Four variants were in Hardy-Weinberg equilibrium in the control group (data not shown, $\mathrm{p}>0.05$ ). There were significant differences in PCV the allele distributions of rs547154 (C2 gene) and rs2072633 (CFB gene) between the PCV group and the controls. The RAP allele distribution of rs2072633 differed significantly between the RAP group and the controls. The tAMD group showed no difference from the controls.

The results of logistic regression analysis, with adjustment for confounding factors, including age, gender and risk factors, are shown in Table 3. This analysis was performed for the dominant or recessive genotype models showing significant results, as presented in

Table 1 Characteristics of study participants

\begin{tabular}{|c|c|c|c|c|c|c|c|c|c|}
\hline & \multicolumn{8}{|c|}{ Case } & \multirow{2}{*}{ Control } \\
\hline & Total AMD & P vs. control & Typical AMD & P vs. control & PCV & P vs. control & RAP & P vs. control & \\
\hline Subjects, n & 677 & & 250 & & 376 & & 51 & & 274 \\
\hline Male/female & $472 / 205$ & $<0.0001^{*}$ & $187 / 63$ & $<0.0001^{*}$ & $266 / 110$ & $<0.0001^{*}$ & $19 / 32$ & 0.757 & $110 / 164$ \\
\hline Age & $72.1( \pm 8.7)$ & 0.157 & $73.6( \pm 7.5)$ & 0.289 & $70.0( \pm 8.9)$ & $<0.0001^{*}$ & $80.9( \pm 6.8)$ & $<0.0001^{*}$ & $72.9( \pm 7.4)$ \\
\hline HT & $39 \%$ & 0.308 & $41 \%$ & 0.658 & $38 \%$ & 0.226 & $41 \%$ & 0.878 & $43 \%$ \\
\hline DM & $11 \%$ & $<0.0001^{*}$ & $14 \%$ & 0.079 & $9 \%$ & 0.081 & $6 \%$ & $0.016^{*}$ & $20 \%$ \\
\hline Smoker & $35 \%$ & $<0.0001^{*}$ & $37 \%$ & $<0.0001^{*}$ & $36 \%$ & $<0.0001^{*}$ & $16 \%$ & $84 \%$ & $18 \%$ \\
\hline
\end{tabular}

p-values reflect comparisons between each of the case groups and the control group, calculated using Fisher's exact test. ${ }^{*} \mathrm{p}<0.05$. 
Table 2 Genotype and allele distributions in AMD patients and control group

\begin{tabular}{|c|c|c|c|c|c|c|c|c|c|c|c|c|c|c|c|c|c|}
\hline & & & & \multicolumn{14}{|c|}{ Total } \\
\hline & & & & \multicolumn{3}{|c|}{ Total AMD patients } & \multicolumn{3}{|c|}{ tAMD } & \multicolumn{3}{|c|}{ PCV } & \multicolumn{3}{|c|}{ RAP } & \multicolumn{2}{|c|}{ Control } \\
\hline & & & & $n$ & $\%$ & $p$-value & $\mathrm{n}$ & $\%$ & p-value & $\mathrm{n}$ & $\%$ & p-value & $\mathrm{n}$ & $\%$ & $\overline{p \text {-value }}$ & $\mathrm{n}$ & $\%$ \\
\hline \multirow[t]{8}{*}{ rs547154 } & Genotype & & $\mathrm{G} / \mathrm{G}$ & 601 & $88.8 \%$ & 0.024 & 221 & $88.4 \%$ & 0.192 & 335 & $89.1 \%$ & 0.053 & 45 & $88.2 \%$ & 0.561 & 229 & $83.6 \%$ \\
\hline & & & $\mathrm{T} / \mathrm{G}$ & 74 & $10.9 \%$ & & 28 & $11.2 \%$ & & 40 & $10.6 \%$ & & 6 & $11.8 \%$ & & 41 & $15.0 \%$ \\
\hline & & & $T / T$ & 2 & $0.3 \%$ & & 1 & $0.4 \%$ & & 1 & $0.3 \%$ & & 0 & $0.0 \%$ & & 4 & $1.5 \%$ \\
\hline & & Dominant model & $\mathrm{G} / \mathrm{G}$ & 601 & $88.8 \%$ & 0.032 & 221 & $88.4 \%$ & 0.132 & 335 & $89.1 \%$ & 0.046 & 45 & $88.2 \%$ & 0.530 & 229 & $83.6 \%$ \\
\hline & & & $\mathrm{TG}+\pi$ & 76 & $11.2 \%$ & & 29 & $11.6 \%$ & & 41 & $10.9 \%$ & & 6 & $11.8 \%$ & & 45 & $16.4 \%$ \\
\hline & & Recessive model & $\pi$ & 2 & $0.3 \%$ & 0.061 & 1 & $0.4 \%$ & 0.375 & 1 & $0.3 \%$ & 0.168 & 0 & $0.0 \%$ & 0.385 & 4 & $1.5 \%$ \\
\hline & Allele & & G & 1276 & $94.2 \%$ & $0.004^{*}$ & 470 & $94.0 \%$ & 0.026 & 710 & $94.4 \%$ & $0.005^{*}$ & 96 & $94.1 \%$ & 0.260 & 499 & $91.1 \%$ \\
\hline & & & T & 78 & $5.8 \%$ & & 30 & $6.0 \%$ & & 42 & $5.6 \%$ & & 6 & $5.9 \%$ & & 49 & $8.9 \%$ \\
\hline \multirow[t]{9}{*}{ rs541862 } & Genotype & & $T / T$ & 600 & $88.6 \%$ & 0.054 & 221 & $88.4 \%$ & 0.246 & 335 & $89.1 \%$ & 0.078 & 44 & $86.3 \%$ & 0.715 & 229 & $83.6 \%$ \\
\hline & & & $\mathrm{T} / \mathrm{C}$ & 75 & $11.1 \%$ & & 28 & $11.2 \%$ & & 40 & $10.6 \%$ & & 7 & $13.7 \%$ & & 42 & $15.3 \%$ \\
\hline & & & $\mathrm{C} / \mathrm{C}$ & 2 & $0.3 \%$ & & 1 & $0.4 \%$ & & 1 & $0.3 \%$ & & 0 & $00.0 \%$ & & 3 & $1.1 \%$ \\
\hline & & Dominant model & $\mathrm{T} / \mathrm{T}$ & 600 & $88.6 \%$ & 0.038 & 221 & $88.4 \%$ & 0.132 & 335 & $89.1 \%$ & 0.046 & 44 & $86.3 \%$ & 0.835 & 229 & $83.6 \%$ \\
\hline & & & $\mathrm{TC}+\mathrm{CC}$ & 77 & $11.4 \%$ & & 29 & $11.6 \%$ & & 41 & $10.9 \%$ & & 7 & $13.7 \%$ & & 45 & $16.4 \%$ \\
\hline & & Recessive model & $\mathrm{C} / \mathrm{C}$ & 2 & $0.3 \%$ & 0.147 & 1 & $0.4 \%$ & 0.625 & 1 & $0.3 \%$ & 0.315 & $00.0 \%$ & 0.453 & 3 & $1.1 \%$ & \\
\hline & & & $\mathrm{TC}+\pi$ & 675 & $99.7 \%$ & & 249 & $99.6 \%$ & & 375 & $99.7 \%$ & 51 & & $100.0 \%$ & & 271 & $98.9 \%$ \\
\hline & Allele & & T & 1275 & $94.2 \%$ & 0.025 & 470 & $94.0 \%$ & 0.099 & 710 & $94.4 \%$ & 0.027 & 95 & $93.1 \%$ & 0.698 & 500 & $91.2 \%$ \\
\hline & & & C & 79 & $5.8 \%$ & & 30 & $6.0 \%$ & & 42 & $5.6 \%$ & & 7 & $6.9 \%$ & & 48 & $8.8 \%$ \\
\hline \multirow[t]{9}{*}{ rs2072633 } & rs2072633 & & $\mathrm{G} / \mathrm{G}$ & 115 & $17.0 \%$ & $0.0024^{*}$ & 48 & $19.2 \%$ & 0.120 & 61 & $16.2 \%$ & $0.0026^{*}$ & 6 & $11.8 \%$ & 0.048 & 69 & $25.2 \%$ \\
\hline & & & $\mathrm{G} / \mathrm{A}$ & 323 & $47.7 \%$ & & 120 & $48.0 \%$ & & 178 & $47.3 \%$ & & 25 & $49.0 \%$ & & 134 & $48.9 \%$ \\
\hline & & & A/A & 239 & $35.3 \%$ & & 82 & $32.8 \%$ & & 137 & $36.4 \%$ & & 20 & $39.2 \%$ & & 71 & $25.9 \%$ \\
\hline & & Dominant model & $\mathrm{G} / \mathrm{G}$ & 115 & $17.0 \%$ & $0.0038^{*}$ & 48 & $19.2 \%$ & 0.101 & 61 & $16.2 \%$ & $0.0048^{*}$ & 6 & $11.8 \%$ & 0.037 & 69 & $25.2 \%$ \\
\hline & & & $G A+A A$ & 562 & $83.0 \%$ & & 202 & $80.8 \%$ & & 315 & $83.8 \%$ & & 45 & $88.2 \%$ & & 205 & $74.8 \%$ \\
\hline & & Recessive model & A/A & 239 & $35.3 \%$ & $0.0051^{*}$ & 82 & $32.8 \%$ & 0.083 & 137 & $36.4 \%$ & $0.0045^{*}$ & 20 & $39.2 \%$ & 0.052 & 71 & $25.9 \%$ \\
\hline & & & $\mathrm{GA}+\mathrm{GG}$ & 438 & $64.7 \%$ & & 168 & $67.2 \%$ & & 239 & $63.6 \%$ & & 31 & $60.8 \%$ & & 203 & $74.1 \%$ \\
\hline & Allele & & G & 553 & $40.8 \%$ & $0.0005^{*}$ & 216 & $43.2 \%$ & 0.037 & 300 & $39.9 \%$ & $0.0005^{*}$ & 37 & $36.3 \%$ & 0.013 & 272 & $49.6 \%$ \\
\hline & & & A & 801 & $59.2 \%$ & & 284 & $56.8 \%$ & & 452 & $60.1 \%$ & & 65 & $63.7 \%$ & & 276 & $50.4 \%$ \\
\hline \multirow[t]{5}{*}{ rs4151667 } & Genotype & & $\mathrm{T} / \mathrm{T}$ & 653 & $96.5 \%$ & 0.386 & 241 & $96.4 \%$ & 0.514 & 363 & $96.5 \%$ & 0.412 & 49 & $96.1 \%$ & 0.797 & 261 & $95.3 \%$ \\
\hline & & & $\mathrm{A} / \mathrm{T}$ & 24 & $3.5 \%$ & & 9 & $3.6 \%$ & & 13 & $3.5 \%$ & & 2 & $3.9 \%$ & & 13 & $4.7 \%$ \\
\hline & & & $\mathrm{A} / \mathrm{A}$ & 0 & $0.0 \%$ & & 0 & $0.0 \%$ & & 0 & $0.0 \%$ & & 0 & $0.0 \%$ & & 0 & $0.0 \%$ \\
\hline & & Dominant mode & $\mathrm{T} / \mathrm{T}$ & 653 & $96.5 \%$ & 0.459 & 241 & $96.4 \%$ & 0.664 & 363 & $96.5 \%$ & 0.424 & 49 & $96.1 \%$ & 0.797 & 261 & $95.3 \%$ \\
\hline & & & $A T+A A$ & 24 & $3.5 \%$ & & 9 & $3.6 \%$ & & 13 & $3.5 \%$ & & 2 & $3.9 \%$ & & 13 & $4.7 \%$ \\
\hline
\end{tabular}


Table 2 Genotype and allele distributions in AMD patients and control group (Continued)

\begin{tabular}{|c|c|c|c|c|c|c|c|c|c|c|c|c|c|c|c|c|}
\hline & Recessive model & $\mathrm{A} / \mathrm{A}$ & 0 & $0.0 \%$ & - & 0 & $0.0 \%$ & - & 0 & $0.0 \%$ & - & 0 & $0.0 \%$ & - & 0 & $0.0 \%$ \\
\hline & & $A T+\pi$ & 677 & $100.0 \%$ & & 250 & $100.0 \%$ & & 376 & $100.0 \%$ & & 51 & $100.0 \%$ & & 274 & $100.0 \%$ \\
\hline \multirow[t]{2}{*}{ Allele } & & T & 1330 & $98.2 \%$ & 0.461 & 491 & $98.2 \%$ & 0.667 & 739 & $98.3 \%$ & 0.429 & 100 & $98.0 \%$ & 0.779 & 535 & $97.6 \%$ \\
\hline & & A & 24 & $1.8 \%$ & & 9 & $1.8 \%$ & & 13 & $1.7 \%$ & & 2 & $2.0 \%$ & & 13 & $2.4 \%$ \\
\hline
\end{tabular}

AMD; age related macular degeneration tAMD; typical age related macular degeneration PCV; polypoidal choroidal vasculopathy RAP; retinal angiomatous proliferation.

$P$ - values are for the comparison between cases and controls.

$p$ - values for genotypes were calculated by Fisher's exact test. (after Bonferroni correction * $p<0.0125$ ). 
Table 3 Logistic regression analysis with adjustment for confounding factors

\begin{tabular}{|c|c|c|c|c|c|c|c|c|c|c|c|c|c|c|c|c|c|}
\hline & & \multicolumn{4}{|c|}{ Total AMD patients } & \multicolumn{4}{|c|}{ tAMD } & \multicolumn{4}{|c|}{ PCV } & \multicolumn{4}{|c|}{ RAP } \\
\hline & & $\begin{array}{c}p \text {-value } \\
\text { vs. control }\end{array}$ & $\begin{array}{l}\text { (Bonferroni } \\
\text { correction) }\end{array}$ & OR & $95 \% \mathrm{Cl}$ & $\begin{array}{c}p \text {-value } \\
\text { vs. control }\end{array}$ & $\begin{array}{l}\text { (Bonferroni } \\
\text { correction) }\end{array}$ & OR & $95 \% \mathrm{Cl}$ & $\begin{array}{c}\text { p-value } \\
\text { vs. control }\end{array}$ & $\begin{array}{l}\text { (Bonferroni } \\
\text { correction) }\end{array}$ & OR & $95 \% \mathrm{Cl}$ & $\begin{array}{c}p \text {-value } \\
\text { vs. control }\end{array}$ & $\begin{array}{l}\text { (Bonferroni } \\
\text { correction) }\end{array}$ & OR & $95 \% \mathrm{Cl}$ \\
\hline \multirow[t]{2}{*}{ rs547154 } & dominant model & $0.044^{*}$ & 0.176 & 0.62 & $0.39-0.99$ & 0.186 & 0.744 & & & 0.103 & 0.412 & & & 0.626 & 1.000 & & \\
\hline & recessive model & 0.051 & 0.204 & & & 0.159 & 0.636 & & & 0.105 & 0.420 & & & - & & & \\
\hline \multirow[t]{2}{*}{ rs541862 } & dominant model & $0.049^{*}$ & 0.196 & 0.63 & $0.39-0.99$ & 0.169 & 0.676 & & & 0.119 & 0.476 & & & 0.877 & 1.000 & & \\
\hline & recessive model & 0.133 & 0.532 & & & 0.232 & 0.928 & & & 0.213 & 0.852 & & & 0.996 & 1.000 & & \\
\hline \multirow[t]{2}{*}{ rs2072633 } & dominant model & $0.0003^{*}$ & $0.001 * *$ & 0.49 & $0.33-0.73$ & $0.042^{*}$ & 0.168 & 0.59 & $0.36-0.99$ & $0.001^{*}$ & $0.004^{* *}$ & & & $0.048^{*}$ & 0.192 & 0.35 & $0.13-0.99$ \\
\hline & recessive model & $0.031^{*}$ & 0.124 & 0.68 & $0.48-0.97$ & 0.312 & 1 & & & $0.010^{*}$ & $0.04^{* *}$ & 0.46 & $0.29-0.74$ & 0.423 & 1.000 & & \\
\hline \multirow[t]{2}{*}{ rs4151667 } & dominant model & 0.273 & 1 & & & 0.952 & 1 & & & 0.270 & 1 & 0.59 & $0.40-0.88$ & 0.769 & 1.000 & & \\
\hline & recessive model & - & & & & - & & & & - & & & & - & & & \\
\hline
\end{tabular}

Logistic regression analysis was performed for each genotype with adjustment for confounding factors (age, gender, hypertension, diabetes mellitus and smoking).

$\mathrm{PCV}$; polypoidal choroidal vasculopathy.

$\mathrm{OR}$; odds ratios $\mathrm{Cl}$; confidence intervals.

$\mathrm{p}$-values are for the comparisons between cases and controls.

$p$-values are for the comparisons between cases and controls.
p-values for genotypes were calculated using Fisher's exact test. ${ }^{*} p<0.05 B$.

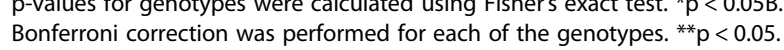

Blanks indicate that there were no siginificant differences. 
Table 2. Susceptibility genotypes were those with high frequencies in patient groups in case-control studies. The rs2072633 distribution of the controls differed significantly from those of the tAMD, PCV and RAP groups. After Bonferroni correction, only PCV showed significant difference in this SNP.

The clinical features of $\mathrm{PCV}$ patients and the control group are shown in Table 4 . There were significant differences in polyp numbers and GLD, both of which were greater in polypoidal CNV group.

Distributions of genotypes and alleles of the four variants are shown in Table 5. Four variants were in Hardy-Weinberg equilibrium in the control group (data not shown, $\mathrm{p}>0.05$ ). There were significant differences in all genotype models and allele distributions of rs547154 (C2 gene), rs541862 and rs2072633 (CFB gene), but not rs4151667, between the polypoidal CNV group and the controls. However, there were no significant differences in any genotype model or allele distribution for any of the SNPs between the typical PCV and control groups.

The results of logistic regression analysis, with adjustment for confounding factors, including age, gender and risk factors, are shown in Tables 6 and 7. This analysis was performed for the dominant or recessive genotype models showing significant results, as presented in Table 5. Susceptibility genotypes were those with high frequencies in patient groups in case-control studies. The distributions of rs541862, rs547154 and rs2072633 differed significantly between the controls and the polypoidal CNV group. After Bonferroni correction, the distribution of rs2072633 remained significant only for polypoidal $\mathrm{CNV}$, i.e. not for typical PCV. Logistic regression analysis was also performed to compare the polypoidal CNV and typical PCV groups. The only significant difference, after adjusting for confounding factors such as polyp numbers and GLD, was in rs2072633. After Bonferroni correction, no significant difference remained.

LD was assessed for three SNPs in CFB, and the distribution of estimated haplotype frequencies is shown in Tables 8 and 9. The T-A-T(rs541862-rs2072633rs4151667) and C-G-T haplotypes both showed strong associations in the polypoidal $\mathrm{CNV}$, typical $\mathrm{PCV}$ and control groups. Furthermore, the T-A-A haplotype differed significantly between polypoidal $\mathrm{CNV}$ and typical PCV.

\section{Discussion}

ARMS2 genes, especially the rs10490924 of CFH and rs1061170, are both known as PCV susceptibility genes $[24,25]$. On the other hand, our group previously reported that typical PCV did not correlate significantly with rs10490924 [19]. This result raised the possibility of two distinct genetic types of $\mathrm{PCV}$. In the present study, the $\mathrm{C} 2$ gene and the CFB gene were also found to be associated with polypoidal CNV, in terms of both genotypes and allele distributions. No associations with typical PCV were detected. These results indicate the $\mathrm{C} 2$ and $\mathrm{CFB}$ genes to also be associated with PCV subtypes. Our group recently reported typical PCV to have the features of abnormal choroidal vessels and that polypoidal $\mathrm{CNV}$ also has features of neovascularization. The differences between $\mathrm{AAMD}$ and polypoidal CNV were that the latter had polypoidal lesion detectable by IA, while tAMD had no polypoidal lesion. Furthermore, polypoidal CNV is characterized by a larger GLD and more polyps than typical PCV [18]. As polypoidal CNV has neovascularization features, the ARMS2 gene might be highly associated with neovascularization. Though there are reports describing rs4151667 as being associated with AMD, the minor allele homozygous frequency was very low in all of these reports $[9,10]$. In this

Table 4 Characteristics of PCV participants

\begin{tabular}{|c|c|c|c|c|c|c|c|c|}
\hline & \multicolumn{7}{|c|}{ Case } & \multirow{2}{*}{ Control } \\
\hline & Total PCV & P vs. control & Polypoidal CNV & P vs. control & P vs. typical PCV & Typical PCV & P vs. control & \\
\hline Subjects, n & 282 & & 84 & & & 198 & & 274 \\
\hline Male/female & $195 / 87$ & $<0.0001^{*}$ & $63 / 21$ & $<0.0001^{*}$ & 0.205 & $132 / 66$ & $<0.0001^{*}$ & $110 / 164$ \\
\hline $\mathrm{Age}( \pm \mathrm{SD})$ & $70.0( \pm 8.7)$ & $<0.0001^{*}$ & $68.8( \pm 8.9)$ & $<0.0001^{*}$ & 0.130 & $70.5( \pm 8.7)$ & $<0.0001^{*}$ & $72.9( \pm 7.4)$ \\
\hline Hypertension & $39 \%$ & 0.390 & $38 \%$ & 0.45 & 0.792 & $40 \%$ & 0.509 & $43 \%$ \\
\hline Diabetes & $9 \%$ & $<0.0001^{*}$ & $10 \%$ & $0.032^{*}$ & 0.649 & $8 \%$ & $<0.0001^{*}$ & $20 \%$ \\
\hline Smoking & $33 \%$ & $<0.0001^{*}$ & $37 \%$ & $<0.0001^{*}$ & 0.406 & $31 \%$ & $0.001^{*}$ & $18 \%$ \\
\hline Number of polyps & - & & 4.17 & & $<0.0001^{*}$ & 1.95 & & - \\
\hline GLD, mm & - & & 3.78 & & $<0.0001^{*}$ & 2.78 & & - \\
\hline
\end{tabular}

p-values reflect comparisons between each of the case groups and the control group, calculated using Fisher's exact test * $\mathrm{p}<0.05$. PCV; polypoidal choroidal vasculopathy CNV; choroidal neovascularization GLD; greatest linear dimension SD; standard deviation. 
Table 5 Genotype and allele distributions in PCV patients and control group

\begin{tabular}{|c|c|c|c|c|c|c|c|c|c|c|c|c|c|}
\hline & & & \multicolumn{3}{|c|}{ Total PCV patients } & \multicolumn{3}{|c|}{ Polypoidal CNV } & \multicolumn{3}{|c|}{ Typical PCV } & \multicolumn{2}{|c|}{ Control } \\
\hline & & & Number & $\%$ & p-value & Number & $\%$ & p-value & Number & $\%$ & p-value & Number & $\%$ \\
\hline \multirow[t]{9}{*}{ rs547154 } & Genotype & $\mathrm{G} / \mathrm{G}$ & 255 & $90.4 \%$ & & 80 & $95 \%$ & & 175 & $88 \%$ & & 229 & $84 \%$ \\
\hline & & $\mathrm{T} / \mathrm{G}$ & 26 & $9.2 \%$ & 0.0400 & 4 & $5 \%$ & 0.023 & 22 & $11 \%$ & 0.276 & 41 & $15 \%$ \\
\hline & & $\mathrm{T} / \mathrm{T}$ & 1 & $0.4 \%$ & & 0 & $0 \%$ & & 1 & $1 \%$ & & 4 & $1 \%$ \\
\hline & Dominant model & $\mathrm{G} / \mathrm{G}$ & 255 & $90.4 \%$ & 0.016 & 80 & $95 \%$ & $0.007^{*}$ & 175 & $88 \%$ & 0.142 & 229 & $84 \%$ \\
\hline & & $\mathrm{TG}+\pi$ & 27 & $9.6 \%$ & & 4 & $5 \%$ & & 23 & $12 \%$ & & 45 & $16 \%$ \\
\hline & Recessive model & $\pi$ & 1 & $0.4 \%$ & 0.168 & 0 & $0 \%$ & 0.265 & 1 & $1 \%$ & 0.317 & 4 & $1 \%$ \\
\hline & & $\mathrm{TG}+\mathrm{GG}$ & 281 & $99.6 \%$ & & 84 & $100 \%$ & & 197 & $99 \%$ & & 270 & $99 \%$ \\
\hline & Allele & G & 536 & $95.0 \%$ & $0.009^{*}$ & 164 & $98 \%$ & $0.004^{*}$ & 372 & $94 \%$ & 0.110 & 499 & $91 \%$ \\
\hline & & $\mathrm{T}$ & 28 & $5.0 \%$ & & 4 & $2 \%$ & & 24 & $6 \%$ & & 49 & $9 \%$ \\
\hline \multirow[t]{9}{*}{ rs541862 } & Genotype & $\mathrm{T} / \mathrm{T}$ & 255 & $90.4 \%$ & & 80 & $95 \%$ & & 175 & $88 \%$ & & 229 & $84 \%$ \\
\hline & & $\mathrm{T} / \mathrm{C}$ & 26 & $9.2 \%$ & 0.049 & 4 & $5 \%$ & 0.023 & 22 & $11 \%$ & 0.318 & 42 & $15 \%$ \\
\hline & & $\mathrm{C} / \mathrm{C}$ & 1 & $0.4 \%$ & & 0 & $0 \%$ & & 1 & $1 \%$ & & 3 & $1 \%$ \\
\hline & Dominant model & $\mathrm{T} / \mathrm{T}$ & 255 & $90.4 \%$ & 0.016 & 80 & $95 \%$ & $0.007^{*}$ & 175 & $88 \%$ & 0.142 & 229 & $84 \%$ \\
\hline & & $\mathrm{TC}+\mathrm{CC}$ & 27 & $9.6 \%$ & & 4 & $5 \%$ & & 23 & $12 \%$ & & 45 & $16 \%$ \\
\hline & Recessive model & $\mathrm{C} / \mathrm{C}$ & 1 & $0.4 \%$ & 0.302 & 0 & $0 \%$ & 0.336 & 1 & $1 \%$ & 0.490 & 3 & $1 \%$ \\
\hline & & $\mathrm{TC}+\pi \mathrm{T}$ & 281 & $99.6 \%$ & & 84 & $100 \%$ & & 197 & $99 \%$ & & 271 & $99 \%$ \\
\hline & Allele & $\mathrm{T}$ & 536 & $95.0 \%$ & 0.013 & 164 & $98 \%$ & $0.004^{*}$ & 372 & $94 \%$ & 0.137 & 500 & $91 \%$ \\
\hline & & C & 28 & $5.0 \%$ & & 4 & $2 \%$ & & 24 & $6 \%$ & & 48 & $9 \%$ \\
\hline \multirow[t]{9}{*}{ rs2072633 } & Genotype & $\mathrm{G} / \mathrm{G}$ & 50 & $17.7 \%$ & & 13 & $15 \%$ & & 37 & $19 \%$ & & 69 & $25 \%$ \\
\hline & & $\mathrm{G} / \mathrm{A}$ & 131 & $46.5 \%$ & 0.017 & 34 & $40 \%$ & $0.005^{*}$ & 97 & $49 \%$ & 0.149 & 134 & $49 \%$ \\
\hline & & A/A & 101 & $35.8 \%$ & & 37 & $44 \%$ & & 64 & $32 \%$ & & 71 & $26 \%$ \\
\hline & Dominant model & $\mathrm{G} / \mathrm{G}$ & 50 & $17.7 \%$ & 0.032 & 13 & $15 \%$ & 0.064 & 37 & $19 \%$ & 0.095 & 69 & $25 \%$ \\
\hline & & $\mathrm{GA}+\mathrm{AA}$ & 232 & $82.3 \%$ & & 71 & $85 \%$ & & 161 & $81 \%$ & & 205 & $75 \%$ \\
\hline & Recessive model & $\mathrm{A} / \mathrm{A}$ & 101 & $35.8 \%$ & $0.012^{*}$ & 37 & $44 \%$ & $0.002^{*}$ & 64 & $32 \%$ & 0.128 & 71 & $26 \%$ \\
\hline & & $\mathrm{GA}+\mathrm{GG}$ & 181 & $64.2 \%$ & & 47 & $56 \%$ & & 134 & $68 \%$ & & 203 & $74 \%$ \\
\hline & Allele & G & 231 & $41.0 \%$ & $0.004^{*}$ & 60 & $36 \%$ & $0.002^{*}$ & 171 & $43 \%$ & 0.055 & 272 & $50 \%$ \\
\hline & & A & 333 & $59.0 \%$ & & 108 & $64 \%$ & & 225 & $57 \%$ & & 276 & $50 \%$ \\
\hline \multirow[t]{9}{*}{ rs4151667 } & Genotype & $\mathrm{T} / \mathrm{T}$ & 273 & $96.8 \%$ & & 81 & $96 \%$ & & 192 & $97 \%$ & & 261 & $95 \%$ \\
\hline & & $\mathrm{A} / \mathrm{T}$ & 9 & $3.2 \%$ & 0.348 & 3 & $4 \%$ & 0.649 & 6 & $3 \%$ & 0.350 & 13 & $5 \%$ \\
\hline & & A/A & 0 & $0.0 \%$ & & 0 & $0 \%$ & & 0 & $0 \%$ & & 0 & $0 \%$ \\
\hline & Dominant model & $\mathrm{T} / \mathrm{T}$ & 273 & $96.8 \%$ & 0.348 & 81 & $96 \%$ & 0.649 & 192 & $97 \%$ & 0.350 & 261 & $95 \%$ \\
\hline & & $A T+A A$ & 9 & $3.2 \%$ & & 3 & $4 \%$ & & 6 & $3 \%$ & & 13 & $5 \%$ \\
\hline & Recessive model & A/A & 0 & $0.0 \%$ & - & 0 & $0 \%$ & - & 0 & $0 \%$ & - & 0 & $0 \%$ \\
\hline & & $A T+T T$ & 282 & $100.0 \%$ & & 84 & $100 \%$ & & 198 & $100 \%$ & & 274 & $100 \%$ \\
\hline & Allele & $\mathrm{T}$ & 555 & $98.4 \%$ & 0.394 & 165 & $98 \%$ & 0.775 & 390 & $98 \%$ & 0.482 & 535 & $98 \%$ \\
\hline & & A & 9 & $1.6 \%$ & & 3 & $2 \%$ & & 6 & $2 \%$ & & 13 & $2 \%$ \\
\hline
\end{tabular}

PCV; polypoidal choroidal vasculopathy CNV; choroidal neovascularization.

$p$ - values are for the comparison between cases and controls.

$p$ - values for genotypes were calculated by Fisher's exact test. (after Bonferroni correction ${ }^{*} p<0.0125$ ).

study, the minor allele homozygous frequency of rs 4151667 was zero, such that there was no difference between cases and controls.

Nakata et al. reported the $\mathrm{C} 2$ (rs547154) and CFB (rs541862) genes to be significantly associated with both
tAMD and PCV in the Japanese population [13]. Nevertheless, rs2072633 (CFB gene) and rs4151672 (CFB gene) showed no correlations with either tAMD or PCV. In the present study, we showed rs2072633 to be significantly associated with PCV. This result indicates the 
Table 6 Logistic regression analysis between cases and controls

\begin{tabular}{|c|c|c|c|c|c|c|c|c|c|c|c|c|c|}
\hline & & \multicolumn{4}{|c|}{ Total PCV patients } & \multicolumn{4}{|c|}{ Polypoidal CNV } & \multicolumn{4}{|c|}{ Typical PCV } \\
\hline & & $\begin{array}{c}\text { p-value } \\
\text { vs. control }\end{array}$ & $\begin{array}{l}\text { (Bonferroni } \\
\text { correction) }\end{array}$ & OR & $95 \% \mathrm{Cl}$ & $\begin{array}{c}\text { p-value } \\
\text { vs. Control }\end{array}$ & $\begin{array}{l}\text { (Bonferroni } \\
\text { correction) }\end{array}$ & $O R$ & $95 \% \mathrm{Cl}$ & $\begin{array}{c}\text { p-value } \\
\text { vs. Control }\end{array}$ & $\begin{array}{l}\text { (Bonferroni } \\
\text { correction) }\end{array}$ & $O R$ & $95 \% \mathrm{Cl}$ \\
\hline \multirow[t]{2}{*}{ rs547154 } & dominant model & $0.018^{*}$ & 0.072 & 0.48 & $0.26-0.89$ & $0.014^{*}$ & 0.056 & 0.22 & $0.05-0.86$ & 0.139 & 0.556 & & \\
\hline & recessive model & 0.217 & 0.868 & & & 0.097 & 0.388 & & & 0.409 & 1.000 & & \\
\hline \multirow[t]{2}{*}{ rs541862 } & dominant model & $0.023^{*}$ & 0.092 & 0.49 & $0.26-0.91$ & $0.015^{*}$ & 0.060 & 0.22 & $0.05-0.87$ & 0.162 & 0.648 & & \\
\hline & recessive model & 0.417 & 1 & & & 0.131 & 0.524 & & & 0.646 & 1.000 & & \\
\hline \multirow[t]{2}{*}{ rs2072633 } & dominant model & $0.012^{*}$ & $0.048^{* *}$ & 0.52 & $0.32-0.87$ & 0.104 & 0.416 & & & $0.037^{*}$ & 0.148 & 0.55 & $0.32-0.96$ \\
\hline & recessive model & $0.035^{*}$ & 0.140 & 0.63 & $0.41-0.96$ & $0.009^{*}$ & $0.036^{* *}$ & 0.40 & $0.20-0.79$ & 0.199 & 0.796 & & \\
\hline \multirow[t]{2}{*}{ rs4151667 } & dominant model & 0.326 & 1 & & & 0.984 & 1 & & & 0.211 & 0.844 & & \\
\hline & recessive model & - & - & & & - & - & & & - & & & \\
\hline
\end{tabular}

Logistic regression analysis was performed for each genotype with adjustment for confounding factors (age, gender, hypertension, diabetes mellitus and smoking).

PCV; polypoidal choroidal vasculopathy.

$\mathrm{OR}$; odds ratios $\mathrm{Cl}$; confidence intervals.

$p$-values are for comparisons between cases and controls.

$p$-values for genotypes were calculated using Fisher's exact test. ${ }^{*} p<0.05$.

$p$-values for genotypes were calculated using Fisher's exact test. ${ }^{*} p<0.05$
Bonferroni correction was performed for each of the genotypes. ${ }^{* *} p<0.05$.

Blanks indicate that there were no siginificant differences. 
Table 7 Logistic regression analysis between polypoidal CNV and typical PCV

\begin{tabular}{|c|c|c|c|c|c|}
\hline & & \multicolumn{4}{|c|}{ Polypoidal CNV } \\
\hline & & $p$-value vs. typical PCV & (Bonferroni correction) & OR & $95 \% \mathrm{Cl}$ \\
\hline \multirow[t]{2}{*}{ rs547154 } & dominant model & 0.073 & 0.292 & & \\
\hline & recessive model & 0.392 & 1 & & \\
\hline \multirow[t]{2}{*}{ rs541862 } & dominant model & 0.073 & 0.292 & & \\
\hline & recessive model & 0.392 & 1 & & \\
\hline \multirow[t]{2}{*}{ rs2072633 } & dominant model & 0.720 & 1 & & \\
\hline & recessive model & $0.038^{*}$ & 0.152 & 2.09 & $1.04-4.22$ \\
\hline \multirow[t]{2}{*}{ rs4151667 } & dominant model & 0.915 & 1 & & \\
\hline & recessive model & - & - & & \\
\hline
\end{tabular}

Logistic regression analysis was performed for each genotype with adjustment for confounding factors (age, gender, hypertension, diabetes mellitus and smoking).

$\mathrm{OR}$; odds ratios $\mathrm{Cl}$; confidence intervals GLD; greatest linear dimension. $p$-values are for the comparisons between polypoidal CNV and typical PCV. $p$-values for genotypes were calculated using Fisher's exact test. ${ }^{*} p<0.05$. Bonferroni correction were performed for each genotypes. $p<0.05$.

Blanks indicate that there were no siginificant differences.

CFB genes to be associated with PCV. Before Bonferroni correction, tAMD was also associated with rs547154 and rs2072633. We previously reported that polypoidal CNV resembles tAMD, while typical PCV clearly differs from $\mathrm{CNV}$. Though not significant after Bonferroni correction, given the prior reports dividing $\mathrm{PCV}$ into two types, we can reasonably speculate that the $\mathrm{C} 2$ and $\mathrm{CFB}$ genes might be related to tAMD and polypoidal $\mathrm{CNV}$ but not to typical PCV. The present $\mathrm{C} 2$ and $\mathrm{CFB}$ gene results also are not inconsistent with this possibility. Since typical PCV was not associated with any of the SNPs tested, we can also speculate that typical PCV might differ genetically from AMD.

$\mathrm{C} 2$ and CFB functioned as activators of the complement cascade. CFB is localized to the choroidal vasculature and Bruch's membrane [26]. Smailhodzic et al. reported AMD patients to show increased alternative pathway activation and elevated CFB levels [27]. Scholl et al. also showed plasma CFB to be significantly elevated in AMD patients [28]. For these reasons, AMD might be related to CFB.

Recently, Liu et al. reported the C2-CFB-RDBPSKIV2L region of SNPs to be associated only with tAMD, not with PCV. They concluded that the mechanisms underlying the development of tAMD and PCV

Table 8 Linkage disequilibrium map through 3 SNPs in CFB gene

\begin{tabular}{lccc}
\hline & rs541862 & rs2072633 & rs4151667 \\
\hline rs541862 & - & 0.929 & 0.278 \\
rs2072633 & -0.040 & - & 1 \\
rs4151667 & -0.001 & 0.012 & - \\
\hline
\end{tabular}

The upper right shows the $\mathrm{D}^{\prime}$-value, the lower left the $\mathrm{D}$-value. might be different [29]. Nakashizuka et al. reported histopathological characteristics of PCV [17]. In their report, areas of PCV showed little fibrosis or granulation as compared to those with CNV. This might indicate that typical PCV involves less inflammation than CNV. Since polypoidal $\mathrm{CNV}$ has AMD features, $\mathrm{C} 2$ and $\mathrm{CFB}$ might be related only to polypoidal $\mathrm{CNV}$.

The results presented in Table 8 show that three of the SNPs in CFB were in LD block. Haplotypes T-A-T and T-G-T differed significantly between the PCV and control groups. Furthermore, T-A-T would confer a risk for PCV, while T-G-T would be protective against PCV development. We could reasonably draw the same conclusion for haplotypes C-A-T and C-G-T. These results indicate that rs2072633 might be one of the key SNPs favoring PCV development.

There has been controversy regarding the division of PCV into two subtypes. Tsujikawa et al. reported that if there is risk associated with being homozygous for the ARMS2 gene, it would be the larger GLD in PCV [30]. Their report described two types of PCV, with larger GLD and smaller GLD. The aforementioned report by Miki and colleagues presented results very similar to ours, indicating the ARMS2 gene to have no association with typical PCV [21]. These two reports also support the assumption that the ARMS2 gene is unrelated to PCV $[17,18]$. While IA findings of polypoidal CNV appeared to be consistent with $\mathrm{CNV}$, the histopathological and IA features of typical PCV showed choroidal vasculature abnormalities. These observations suggested polypoidal $\mathrm{CNV}$ to be genetically and histopathologically close to tAMD, a representative form of CNV. Furthermore, typical PCV showed no association with CNV. 
Table 9 Haplotype association analysis in cases and controls

\begin{tabular}{|c|c|c|c|c|c|c|}
\hline \multicolumn{7}{|c|}{ Polypoidal CNV vs. control } \\
\hline \multicolumn{3}{|c|}{ Haplotypes } & \multicolumn{2}{|c|}{$\%$} & \multirow[b]{2}{*}{ Chi-Squ } & \multirow[b]{2}{*}{ p-value } \\
\hline rs541862 & rs2072633 & rs4151667 & Polypoidal CNV & Control & & \\
\hline $\mathrm{T}$ & A & $\mathrm{T}$ & $63 \%$ & $42 \%$ & 22.177 & $<0.0001^{*}$ \\
\hline C & A & $\mathrm{T}$ & $0 \%$ & $9 \%$ & 14.9366 & $0.0001^{*}$ \\
\hline $\mathrm{T}$ & G & $\mathrm{T}$ & $35 \%$ & $47 \%$ & 7.9166 & $0.0049^{*}$ \\
\hline C & G & $\mathrm{T}$ & $2 \%$ & $0 \%$ & 13.5581 & $0.0002^{*}$ \\
\hline $\mathrm{T}$ & G & A & $0 \%$ & $2 \%$ & 3.9293 & $0.0475^{*}$ \\
\hline \multicolumn{7}{|c|}{ Typical PCV vs. control } \\
\hline \multicolumn{3}{|c|}{ Haplotypes } & \multicolumn{2}{|c|}{$\%$} & \multirow[b]{2}{*}{ Chi-Square } & \multirow[b]{2}{*}{$\mathrm{p}$-value } \\
\hline rs541862 & rs2072633 & rs4151667 & Typical PCV & Control & & \\
\hline $\mathrm{T}$ & A & $\mathrm{T}$ & $57 \%$ & $42 \%$ & 20.5614 & $<0.0001^{*}$ \\
\hline C & $A$ & $\mathrm{~T}$ & $0 \%$ & $9 \%$ & 34.8144 & $<0.0001^{*}$ \\
\hline $\mathrm{T}$ & G & $\mathrm{T}$ & $37 \%$ & $47 \%$ & 9.3704 & $0.0022^{*}$ \\
\hline C & G & $\mathrm{T}$ & $6 \%$ & $0 \%$ & 33.5324 & $<0.0001^{*}$ \\
\hline \multicolumn{7}{|c|}{ Polypoidal CNV vs. typical PCV } \\
\hline \multicolumn{3}{|c|}{ Haplotypes } & \multicolumn{2}{|c|}{$\%$} & \multirow[b]{2}{*}{ Chi-Squ } & \multirow[b]{2}{*}{$\mathrm{p}$-value } \\
\hline rs541862 & rs2072633 & rs4151667 & Typical PCV & Polypoidal CNV & & \\
\hline $\mathrm{T}$ & A & $\mathrm{T}$ & $57 \%$ & $63 \%$ & 1.5687 & 0.2104 \\
\hline C & A & $\mathrm{T}$ & $0 \%$ & $0 \%$ & 0 & 1 \\
\hline $\mathrm{T}$ & G & $\mathrm{T}$ & $36 \%$ & $33 \%$ & 0.3302 & 0.5656 \\
\hline C & G & $\mathrm{T}$ & $6 \%$ & $2 \%$ & 3.0395 & 0.0813 \\
\hline $\mathrm{T}$ & A & A & $0 \%$ & $2 \%$ & 7.1092 & $0.0077^{*}$ \\
\hline C & A & A & $0 \%$ & $0 \%$ & 0 & 1 \\
\hline $\mathrm{T}$ & G & A & $1 \%$ & $0 \%$ & 2.1402 & 0.1435 \\
\hline C & G & $A$ & $0 \%$ & $0 \%$ & 0 & 1 \\
\hline
\end{tabular}

${ }^{*} p$-value $>0.05$ calculated by chi-square analysis.

The small sample size with only one genotype is the major limitation of this study. Further study is clearly needed.

\section{Conclusion}

The present study is the first to examine the associations between variants in the $\mathrm{C} 2$ and $\mathrm{CFB}$ genes and PCV subtypes. We found the $\mathrm{C} 2$ and $\mathrm{CFB}$ genes to possibly be genetic markers for polypoidal CNV. Furthermore, these variants showed no associations with typical PCV. These results suggest polypoidal $\mathrm{CNV}$ to have a genetic background different from that of typical PCV. Further studies are needed to examine the effects of various treatments on PCV subtypes.

\section{Abbreviations}

PCV: Polypoidal choroidal vasculopathy; CNV: Choroidal neovascularization; C2: Complement component 2; CFB: Complement factor B; AMD: Agerelated macular degeneration; $\mathrm{CFH}$ : Complement factor $\mathrm{H}$; ARMS2: Agerelated maculopathy susceptibility 2; HTRA1: High-temperature requirement factor A1; tAMD: Typical AMD; IA: Indocyanine green angiography; GLD: Greatest linear dimension.
Competing interests

The authors have no competing interests to declare.

Authors' contributions

KT, TN and MY participated in the design of this study. KT and NS participated in the laboratory work. RM and AK were responsible for participants' enrollment. KT performed the statistical analysis and wrote the draft manuscript. All authors read and approved the final manuscript.

\section{Acknowledgments}

We would like to thank all patients who participated in this study. This work was funded in part by the Research Committee on Chorioretinal Degenerations and Optic Atrophy, and by The Ministry of Health and Welfare of Japan (Mitsuko Yuzawa).

\section{Author details}

'Department of Ophthalmology, Nihon University School of Medicine, 1-8-13 Kandasurugadai, Chiyoda-ku, Tokyo 101-8309, Japan. ${ }^{2}$ Department of Pathology and Microbiology, Nihon University School of Medicine, Tokyo, Japan. 


\section{References}

1. Yasuda M, Kiyohara Y, Hata Y, Arakawa S, Yonemoto K, Doi Y, lida M, Ishibashi T: Nine-year incidence and risk factors for age-related macular degeneration in a defined Japanese population the Hisayama study. Ophthalmology 2009, 116:2135-2140. PubMed.

2. Sperduto RD, Hiller R: Systemic hypertension and age-related maculopathy in the Framingham Study. Arch Ophthalmol 1986, 104:216-219. PubMed.

3. Klein R, Klein BE, Linton KL, DeMets DL: The beaver dam eye study: the relation of age-related maculopathy to smoking. Am J Epidemiol 1993, 137:190-200. PubMed.

4. Klein RJ, Zeiss C, Chew EY, Tsai JY, Sackler RS, Haynes C, Henning AK, SanGiovanni JP, Mane SM, Mayne ST, Bracken MB, Ferris FL, Ott J, Barnstable C, Hoh J: Complement factor $\mathrm{H}$ polymorphism in age-related macular degeneration. Science 2005, 308:385-389. PubMed.

5. Rivera A, Fisher SA, Fritsche LG, Keilhauer CN, Lichtner P, Meitinger T, Weber BH: Hypothetical ARMS2 is a second major susceptibility gene for age-related macular degeneration, contributing independently of complement factor $\mathrm{H}$ to disease risk. Hum Mol Genet 2005, 14:3227-3236. PubMed.

6. Dewan A, Liu M, Hartman S, Zhang SS, Liu DT, Zhao C, Tam PO, Chan WM, Lam DS, Snyder M, Barnstable C, Pang CP, Hoh J: HTRA1 promoter polymorphism in wet age-related macular degeneration. Science 2006 314:989-992. PubMed.

7. Yoshida T, DeWan A, Zhang H, Sakamoto R, Okamoto H, Minami M, Obazawa M, Mizota A, Tanaka M, Saito Y, Takagi I, Hoh J, Iwata T: HTRA1 promoter polymorphism predisposes Japanese to age-related macular degeneration. Mol Vis 2007, 13:545-548. PubMed.

8. Gold B, Merriam JE, Zernant J, Hancox LS, Taiber AJ, Gehrs K, Cramer K, Neel J, Bergeron J, Barile GR, Smith RT, AMD Genetics Clinical Study Group, Hageman GS, Dean M, Allikmets R: Variation in factor B (BF) and complement component 2 (C2) genes is associated with age-related macular degeneration. Nat Genet 2006, 38:458-462. PubMed.

9. Lima LH, Schubert C, Ferrara DC, Merriam JE, Imamura Y, Freund KB, Spaide RF, Yannuzzi LA, Allikmets R: Three major loci involved in age-related macular degeneration are also associated with polypoidal choroidal vasculopathy. Ophthalmology 2010, 117:1567-1570. PubMed.

10. Sun C, Zhao $M, L i X: C F B / C 2$ gene polymorphisms and risk of age-related macular degeneration: a systematic review and meta-analysis. Curr Eye Res 2012, 37:259-271. PubMed.

11. Lee KY, Vithana EN, Mathur R, Yong VH, Yeo IY, Thalamuthu A, Lee MW, Koh AH, Lim MC, How AC, Wong DW, Aung T: Association analysis of CFH, C2, BF, and HTRA1 gene polymorphisms in Chinese patients with polypoidal choroidal vasculopathy. Invest Ophthalmol Vis Sci 2008, 49:2613-2619. PubMed.

12. Kondo N, Honda S, Kuno S, Negi A: Role of RDBP and SKIV2L variants in the major histocompatibility complex class III region in polypoidal choroidal vasculopathy etiology. Ophthalmology 2009, 116:1502-1509. PubMed.

13. Nakata I, Yamashiro K, Yamada R, Gotoh N, Nakanishi H, Hayashi H, AkagiKurashige Y, Tsujikawa A, Otani A, Saito M, lida T, Oishi A, Matsuo K, Tajima K, Matsuda F, Yoshimura N: Significance of C2/CFB variants in age-related macular degeneration and polypoidal choroidal vasculopathy in a Japanese population. Invest Ophthalmol Vis Sci 2012, 53:794-798. PubMed.

14. Spaide RF, Yannuzzi LA, Slakter JS, Sorenson J, Orlach DA: Indocyanine green videoangiography of idiopathic polypoidal choroidal vasculopathy. Retina 1995, 15:100-110. PubMed.

15. Takahashi K, Ishibashi T, Ogura Y, Yuzawa M: Classification and diagnostic criteria of age-related macular degeneration; working group for establishing diagnostic criteria for age-related macular degeneration. Nippon Ganka Gakkai Zasshi 2008, 112:1076-1084. in Japanese, with English abstract) [PubMed]

16. Yuzawa M, Mori R, Kawamura A: The origins of polypoidal choroidal vasculopathy. Br J Ophthalmol 2005, 89:602-607. PubMed.

17. Nakashizuka H, Mitsumata M, Okisaka S, Shimada H, Kawamura A, Mori R, Yuzawa M: Clinicopathologic findings in polypoidal choroidal vasculopathy. Invest Ophthalmol Vis Sci 2008, 49:4729-4737. PubMed.

18. Kawamura A, Yuzawa M, Mori R, Haruyama M, Tanaka K: Indocyanine green angiographic and optical coherence tomographic findings support classification of polypoidal choroidal vasculopathy into two types. Acta Ophthalmol 2013, 91:e474-e481. PubMed.

19. Tanaka K, Nakayama T, Mori R, Sato N, Kawamura A, Mizutani Y, Yuzawa M: Associations of complement factor $\mathrm{H}(\mathrm{CFH})$ and age-related maculopathy susceptibility 2(ARMS2) genotypes with subtypes of polypoidal choroidal vasculopathy. Invest Ophthalmol Vis Sci 2011, 52:7441-7444. PubMed.
20. Okubo A, Hirakawa M, Ito M, Sameshima M, Sakamoto T: Clinical features of early and late stage polypoidal choroidal vasculopathy characterized by lesion size and disease duration. Graefes Arch Clin Exp Ophthalmol 2008, 246:491-499. PubMed.

21. Miki A, Honda S, Kondo N, Negi A: The association of age-related maculopathy susceptibility 2(ARMS2) and complement factor $\mathrm{H}(\mathrm{CFH})$ variants with two angiographic subtypes of polypoidal choroidal vasculopathy. Ophthalmic Genet 2013, 34:146-150. PubMed.

22. Mizutani Y, Nakayama T, Asai S: Study on the association between angioid streaks and ABCC6 as the causal gene of pseudoxanthoma elasticum. Int J Biomed Sci 2006, 2:9-14.

23. Sato N, Nakayama T, Mizutani Y, Yuzawa M: Novel mutations of ABCC6 gene in Japanese patients with angioid streaks. Biochem Biophys Res Commun 2009, 380:548-553. PubMed.

24. Gotoh N, Nakanishi H, Hayashi H, Yamada R, Otani A, Tsujikawa A Yamashiro K, Tamura H, Saito M, Saito K, lida T, Matsuda F, Yoshimura N: ARMS2 (LOC387715) variants in Japanese patients with exudative age-related macular degeneration and polypoidal choroidal vasculopathy. Am J Ophthalmol 2009, 147:1037-1041. PubMed.

25. Hayashi H, Yamashiro K, Gotoh N, Nakanishi H, Nakata I, Tsujikawa A, Otani A, Saito M, lida T, Matsuo K, Tajima K, Yamada R, Yoshimura N: CFH and ARMS2 variations in age-related macular degeneration, polypoidal choroidal vasculopathy, and retinal angiomatous proliferation. Invest Ophthalmol Vis Sci 2010, 51:5914-5919. PubMed.

26. Loyet KM, Deforge LE, Katschke KJ Jr, Diehl L, Graham RR, Pao L, Sturgeon L, Lewin-Koh SC, Hollyfield JG, van Lookeren Campagne M: Activation of the alternative complement pathway in vitreous is controlled by genetics in age-related macular degeneration. Invest Ophthalmol Vis Sci 2012, 53:6628-6637. PubMed.

27. Smailhodzic D, Klaver CC, Klevering BJ, Boon CJ, Groenewoud JM, Kirchhof B, Daha MR, den Hollander Al, Hoyng CB: Risk alleles in CFH and ARMS2 are independently associated with systemic complement activation in age related macular degeneration. Ophthalmology 2012, 119:339-346. PubMed.

28. Scholl HP, Charbel Issa P, Walier M, Janzer S, Pollok-Kopp B, Börncke F, Fritsche LG, Chong NV, Fimmers R, Wienker T, Holz FG, Weber BH, Oppermann M: Systemic complement activation in age-related macular degeneration. PLos One 2008, 3:2593. PubMed.

29. Liu K, Chen LJ, Tam PO, Shi Y, Lai TY, Liu DT, Chiang SW, Yang M, Yang Z, Pang CP: Associations of the C2-CFB-RDBP-SKIV2L locus with age-related macular degeneration and polypoidal choroidal vasculopathy. Ophthalmology 2013, 120:837-884. PubMed.

30. Tsujikawa A, Ojima Y, Yamashiro K, Nakata I, Ooto S, Tamura H, Nakanishi H, Hayashi $\mathrm{H}$, Otani A, Yoshimura N: Association of lesion size and visual prognosis to polypoidal choroidal vasculopathy. Am J Ophthalmol 2011, 151:961-972. PubMed.

doi:10.1186/1471-2415-14-83

Cite this article as: Tanaka et al: Associations of complement factor B and complement component 2 genotypes with subtypes of polypoidal choroidal vasculopathy. BMC Ophthalmology 2014 14:83.

\section{Submit your next manuscript to BioMed Central and take full advantage of:}

- Convenient online submission

- Thorough peer review

- No space constraints or color figure charges

- Immediate publication on acceptance

- Inclusion in PubMed, CAS, Scopus and Google Scholar

- Research which is freely available for redistribution 